\title{
Solved and Unsolved Problems
}

\author{
Michael Th. Rassias (University of Zürich, Switzerland)
}

\section{Probability theory is nothing but common sense reduced to calculation.}

Pierre-Simon Laplace (1749-1827)

The column this month is devoted to probability theory. The proposed problems range from basic to fairly demanding so a wide range of our readers should be able to tackle them. As always, there is also a proposed open research problem. The open problem, along with the relevant discussion, is provided by Martin Hairer.

Probability theory traces back to the 16th century, when the Italian polymath Gerolamo Cardano attempted to mathematically analyse games of chance. More specifically, his book about games of chance, published in 1663 (written ca. 1564), contains the first systematic treatqment of probability. Probability theory also traces back to 17th century France, when Blaise Pascal and Pierre de Fermat corresponded about problems of games of chance. In modern mathematics, probability theory is an extremely applicable and versatile field, which is used in a surprisingly broad spectrum of areas, such as weather prediction, medicine/biology, equity trading, machine perception, music, etc.

\section{Six new problems-solutions solicited}

Solutions will appear in a subsequent issue.

197. In a game, a player moves a counter on the integers according to the following rules. During each round, a fair die is thrown. If the die shows " 5 " or " 6 ", the counter is moved up one position and if it shows " 1 " or " 2 ", it is moved down one position. If the die shows " 3 " or " 4 ", the counter is moved up one position if the current position is positive, down one position if the current position is negative and stays at the same position if the current position is 0 . Let $X_{n}$ denote the position of the player after $n$ rounds when starting at $X_{0}=1$. Find the probability $p$ that $\lim X_{n}=+\infty$ and show that $X_{n} / n \rightarrow 1 / 3$ with probability $p$ and $X_{n} / n \rightarrow-1 / 3$ with probability $1-p$.

(Andreas Eberle, Institute for Applied Mathematics, Probability Theory, Bonn, Germany)

198. Let $B:=\left(B_{t}\right)_{t \geq 0}$ be Brownian motion in the complex plane. Suppose that $B_{0}=1$.

(a) Let $T_{1}$ be the first time that $B$ hits the imaginary axis, $T_{2}$ be the first time after $T_{1}$ that $B$ hits the real axis, $T_{3}$ be the first time after $T_{2}$ that $B$ hits the imaginary axis, etc. Prove that, for each $n \geq 1$, the probability that $\left|B_{T_{n}}\right| \leq 1$ is $1 / 2$.

(b) More generally, let $\ell_{n}$ be lines through 0 for $n \geq 1$ such that $1 \notin \ell_{1}$. Let $T_{1}:=\inf \left\{t \geq 0 ; B_{t} \in \ell_{1}\right\}$ and recursively define $T_{n+1}:=\inf \left\{t>T_{n} ; B_{t} \in \ell_{n+1}\right\}$ for $n \geq 1$. Prove that, for each $n \geq 1$, the probability that $\left|B_{T_{n}}\right| \leq 1$ is $1 / 2$.

(c) In the context of part (b), let $\alpha_{n}$ be the smaller of the two angles between $\ell_{n}$ and $\ell_{n+1}$. Show that $\sum_{n=1}^{\infty} \alpha_{n}=\infty$ iff, for all $\epsilon>0$, the probability that $\epsilon \leq\left|B_{T_{n}}\right| \leq 1 / \epsilon$ tends to 0 as $n \rightarrow \infty$. (d) In the context of part (a), show that

$$
\lim _{n \rightarrow \infty} \mathbf{P}\left[\exp \left(-\delta_{n} \sqrt{n}\right) \leq\left|B_{T_{n}}\right| \leq \exp \left(\delta_{n} \sqrt{n}\right)\right]=\int_{-2 \delta / \pi}^{2 \delta / \pi} \frac{e^{-u^{2} / 2}}{\sqrt{2 \pi}} d u
$$

if $\delta_{n} \geq 0$ tend to $\delta \in[0, \infty]$.

(Russell Lyons, Department of Mathematics, Indiana University, USA. [Partially supported by the National Science Foundation under grant DMS-1612363])

199. Suppose that each carioca (native of Rio de Janeiro) likes at least half of the other $2^{23}$ cariocas. Prove that there exists a set $A$ of 1000 cariocas with the following property: for each pair of cariocas in $A$, there exists a distinct carioca who likes both of them.

(Rob Morris, IMPA, Rio de Janeiro, Brazil)

200. Let $X, Y, Z$ be independent and uniformly distributed in $[0,1]$. What is the probability that three sticks of length $X, Y$ and $Z$ can be assembled together to form a triangle?

(Sebastien Vasey, Department of Mathematics, Harvard University, Cambridge, Massachusetts, USA)

201. Suppose that each hour, one of the following four events may happen to a certain type of cell: it may die, it may split into two cells, it may split into three cells or it may remain a single cell. Suppose these four events are equally likely. Start with a population consisting of a single cell. What is the probability that the population eventually goes extinct?

(Sebastien Vasey, Department of Mathematics, Harvard University, Cambridge, Massachusetts, USA)

202. We flip a fair coin repeatedly and record the outcomes.

(1) How many coin flips do we need on average to see three tails in a row?

(2) Suppose that we stop when we first see heads, heads, tails $(\mathrm{H}$, $\mathrm{H}, \mathrm{T})$ or tails, heads, tails ( $\mathrm{T}, \mathrm{H}, \mathrm{T})$ come up in this order on three consecutive flips. What is the probability that we stop at $\mathrm{H}, \mathrm{H}, \mathrm{T}$ ?

(Benedek Valkó, Department of Mathematics, University of Wisconsin Madison, Madison, Wisconsin, USA)

\section{An Open Problem, by Martin Hairer}

(Mathematics Institute, Imperial College London, UK)

Before trying to formulate this open problem, I would like to start by introducing one of the most important objects in probability theory, namely Brownian motion. One way of viewing Brownian motion is as a random variable $B$ taking values in the space $\mathbb{C}$ of continuous functions from $\mathbb{R}$ to $\mathbb{R}$ and satisfying the following two properties.

Claim 1 (i) One has $B(0)=0$ almost surely.

(ii) For any finite sequence of times $\left(t_{1}, \ldots, t_{n}\right)$, the $\mathbb{R}^{n}$-valued random variable $\left(B\left(t_{1}\right), \ldots, B\left(t_{n}\right)\right)$ is a centred Gaussian random variable such that $\mathbf{E}\left(B\left(t_{i}\right)-B\left(t_{j}\right)\right)^{2}=\left|t_{i}-t_{j}\right|$ for any $i, j \in\{1, \ldots, n\}$. 
Why is Brownian motion so important? One reason is that it appears in the following "functional" version of the central limit theorem. Consider a sequence $\left\{\xi_{i}\right\}_{i \in \mathbb{Z}}$ of independent and identically distributed (i.i.d.) random variables with vanishing expectation and unit variance. We use these to define a collection of random variables $S_{n}$ for $n \in \mathbb{Z}$ by specifying that

$$
S_{0}=0, \quad S_{n+1}-S_{n}=\xi_{n} .
$$

The central limit theorem then tells us that, as $n \rightarrow \infty, S_{n} / \sqrt{n}$ converges in law to a standard Gaussian random variable. On the other hand, we can define a random continuous function $S(t)$ by setting $S(n)=S_{n}$ for $n \in \mathbb{Z}$ and by extending this to arguments in $\mathbb{R}$ by linear interpolation. If we rescale this random function appropriately by setting $S^{(N)}(t)=S(N t) / \sqrt{N}$, we obtain the following result [3].

Theorem 1 As $N \rightarrow \infty$, the sequence of $\mathbb{C}$-valued random variables $S^{(N)}$ converges in law to a Brownian motion.

This way of obtaining Brownian motion immediately suggests a number of properties that are not completely obvious at first sight from the definition above, although they can easily be read off property (ii) above. First, since the $\xi_{n}$ are i.i.d., the collection $\tilde{\xi}_{n}=\xi_{n+m}$ is equal in law to the original sequence for every fixed $m \in \mathbb{Z}$. At the level of $S$, this implies that if we define the translation operators

$$
\left(\mathcal{T}_{\tau} S\right)(t)=S(t+\tau)-S(\tau) \quad \text { so that } \quad \mathcal{T}_{\tau} \mathcal{T}_{v}=\mathcal{T}_{\tau+v},
$$

$\mathcal{T}_{\tau} S \stackrel{\text { law }}{=} S$ for every $\tau \in \mathbb{Z}$. Similarly, we can define rescaling operators

$$
\left(\mathcal{S}_{\alpha}^{\lambda} S\right)(t)=\lambda^{-\alpha} S(\lambda t) \quad \text { so that } \mathcal{S}_{\alpha}^{\lambda} \mathcal{S}_{\alpha}^{\mu}=\mathcal{S}_{\alpha}^{\lambda \mu},
$$

as well as $S^{(N)}=\mathcal{S}_{1 / 2}^{N} S$. Finally, we note that since the $\xi_{i}$ are independent, there exists $\delta>0$ such that, conditional on the 'present' $\left\{S(t):\left|t-t_{0}\right| \leq \delta\right\}$, the 'future' $\left\{S(t): t>t_{0}\right\}$ is independent of the 'past' $\left\{S(t): t \leq t_{0}\right\}$ for every $t_{0} \in \mathbb{R}$. This suggests the following.

Proposition 1 Brownian motion satisfies the following properties.

Claim 2 Translation invariance: $\mathcal{T}_{\tau} B \stackrel{\text { law }}{=} B$ for all $\tau \in \mathbb{R}$.

Scale invariance: $\mathcal{S}_{\alpha}^{\lambda} B \stackrel{\text { law }}{=} B$ for $\alpha=1 / 2$ and all $\lambda>0$.

Markov property: For any $t_{0} \in \mathbb{R}$, conditional on $B_{t_{0}},\left\{B(t): t>t_{0}\right\}$ is independent of $\left\{B(t): t \leq t_{0}\right\}$.

As a matter of fact, up to multiplication by a real number, Brownian motion is the only $\mathbb{C}$-valued random variable with these properties that also vanishes at the origin. Furthermore, even if we relax the second condition to allow for values $\alpha \neq 1 / 2$, it remains the case that Brownian motion is the only continuous stochastic process satisfying all of these properties. If we allow for discontinuous processes then we can find other processes satisfying these properties but there are still very "few" of them. More precisely, for each value $\alpha>1 / 2$, there is a process $L_{\alpha}$ (the so-called 'spectrally positive $1 / \alpha$-stable Lévy process') such that every process satisfying the properties of Proposition 1 is of the form $\kappa_{+} L_{\alpha}-\kappa_{-} \tilde{L}_{\alpha}$, where $\kappa_{ \pm}$are two positive numbers and $\tilde{L}_{\alpha}$ is an independent copy of $L_{\alpha}$.

Processes satisfying the three properties of Proposition 1 arise naturally (or rather, in many cases, are conjectured to arise) as scaling limits of various "toy models" of statistical mechanics. In these situations, however, one is typically interested in processes that do not depend on a time parameter but instead on two or more "spatial" parameters. Furthermore, in most known cases, the processes arising in this way are random Schwartz distributions, so that some care has to be taken with the formulation of the Markov property. One formulation is the following.

Definition 1 A random distribution $\eta$ on $\mathbb{R}^{d}$ satisfies the germ Markov property if, for any smooth domain $D$ and any neighbourhood $U$ of $\partial D$, the laws of $\{\eta(\phi): \operatorname{supp} \phi \subset D\}$ and $\{\eta(\phi): \operatorname{supp} \phi \subset$ $\left.D^{c}\right\}$ are independent, conditional on $\{\eta(\phi): \operatorname{supp} \phi \subset U\}$.
For $d>2$, the "free field" is the analogue of Brownian motion and is defined as the random distribution $\eta$ such that all random variables of the type $\eta(\phi)$ with $\phi \in \mathbb{C}_{0}^{\infty}$ are jointly centred Gaussians with covariance given by

$$
\mathbf{E} \eta(\phi) \eta(\psi)=\int_{\mathbb{R}^{d}} \int_{\mathbb{R}^{d}} \phi(x) \psi(y)|x-y|^{2-d} d x d y .
$$

Again, this is translation invariant, has the germ Markov property and is scale invariant with exponent $\alpha=1-\frac{d}{2}$. (A similar object also exists for $d=2$ and is of great interest but the associated notion of "translation invariance" is more involved.) An answer to the following question would be a gigantic breakthrough in probability theory and mathematical physics.

203* Open Problem. For $d \geq 2$ and $\alpha<0$, characterise all (if any) random distributions that are invariant under the Euclidean transformations, scale invariant with exponent $\alpha$ and satisfy the germ Markov property.

Any partial result, including the description of any previously unknown non-Gaussian random distribution with these properties, would be very welcome. Besides the free field, one such random distribution was recently constructed in $d=2$ with exponent $-1 / 8$ as the scaling limit of the Ising model at criticality $[1,2]$. Conformal field theory provides a conjectured characterisation of a whole family of such objects for a range of exponents $\alpha$ in $d=2$ but the case $d \geq 3$ is wide open, even at the conjectural level. Another breakthrough in this direction was the recent characterisation [4] of the "KPZ fixed point", a space-time random function $H$ (in space dimension 1 ) that is translation invariant, has the germ Markov property and is scale invariant in the sense that $\lambda^{-1} H\left(\lambda^{2} x, \lambda^{3} t\right) \stackrel{\text { law }}{=} H(x, t)$.

\section{References}

[1] F. Camia, C. Garben and C. M. Newman. Planar Ising magnetization field I. Uniqueness of the critical scaling limit. Ann. Probab. 43, (2015), 528-571. arXiv:1205.6610. Doi 10.1214/13-AOP881.

[2] F. Camia, C. Garben and C. M. Newman. Planar Ising magnetization field II. Properties of the critical and near-critical scaling limits. Ann. Inst. Henri Poincaré Probab. Stat. 52, (2016), 146-161. arXiv:1307.3926. Doi 10.1214/14-AIHP643.

[3] M.D. Donsker, An invariance principle for certain probability limit theorems. Mem. Amer. Math. Soc. 6 (1951), 12.

[4] K. Matetski, J. Quastel and D. Remenik, The KPZ fixed point. ArXiv e-prints (2017). arXiv: 1701.00018

\section{Solutions}

187. Let $\left(a_{n}\right)_{n \geq 1},\left(b_{n}\right)_{n \geq 1}$ and $\left(c_{n}\right)_{n \geq 0}$ be sequences such that $a_{n}>0, b_{n}>0$ and $c_{n}>0$ for $n \geq 1$ and:

(G1) $c_{0}=0$ and $c_{n}$ is increasing,

(G2) $c_{n+1}-c_{n}$ is decreasing for $n \geq 0$,

(G3) $c_{k}\left(\frac{a_{k+1}}{a_{k}}-1\right) \geq c_{n}\left(\frac{b_{n+1}}{b_{n}}-1\right)$ for $1 \leq k<n$.

Given a function $f$, let

$$
A_{n}=\frac{1}{c_{n-1}} \sum_{k=1}^{n-1} f\left(\frac{a_{k}}{b_{n}}\right), \quad n \geq 2 .
$$

Then, if $f$ is real, convex increasing and non-negative on an interval $[D, E]$ that includes all the points $\frac{a_{k}}{b_{n}}$ for $k<n$, prove that $A_{n}$ increases with $n$.

(Shoshana Abramovich, University of Haifa, Israel) 
Solution by the proposer. Similarly to [1, Theorem 5.1], we will show that under our conditions,

$$
A_{n+1}-A_{n} \geq 0, \quad n \geq 2 .
$$

By the definition of $A_{n}$, we get

$$
\begin{aligned}
A_{n+1}-A_{n} & =\frac{1}{c_{n}} \sum_{k=1}^{n} f\left(\frac{a_{k}}{b_{n+1}}\right)-\frac{1}{c_{n-1}} \sum_{k=1}^{n-1} f\left(\frac{a_{k}}{b_{n}}\right) \\
& =\frac{1}{c_{n-1}}\left[\frac{c_{n-1}}{c_{n}} \sum_{k=1}^{n} f\left(\frac{a_{k}}{b_{n+1}}\right)-\sum_{k=1}^{n-1} f\left(\frac{a_{k}}{b_{n}}\right)\right] .
\end{aligned}
$$

To enable proving (3), we rewrite

$$
\frac{c_{n-1}}{c_{n}} \sum_{k=1}^{n} f\left(\frac{a_{k}}{b_{n+1}}\right), \quad n \geq 2 .
$$

By using (G2), we get that

$$
c_{n-1}>c_{n}-c_{k}+c_{k-1} \text {. }
$$

As $f$ is non-negative and $c_{n}>0$ when $n \geq 1$, we get from (5) that

$$
\begin{aligned}
\frac{c_{n-1}}{c_{n}} \sum_{k=1}^{n} f\left(\frac{a_{k}}{b_{n+1}}\right) & \geq \sum_{k=1}^{n} \frac{c_{k-1}+c_{n}-c_{k}}{c_{n}} f\left(\frac{a_{k}}{b_{n+1}}\right) \\
& =\sum_{k=1}^{n} \frac{c_{k-1}}{c_{n}} f\left(\frac{a_{k}}{b_{n+1}}\right)+\sum_{k=1}^{n} \frac{c_{n}-c_{k}}{c_{n}} f\left(\frac{a_{k}}{b_{n+1}}\right) .
\end{aligned}
$$

It is given that $c_{0}=0$, therefore (6) leads to

$$
\begin{aligned}
\frac{c_{n-1}}{c_{n}} \sum_{k=1}^{n} f\left(\frac{a_{k}}{b_{n+1}}\right) & \geq \sum_{k=1}^{n} \frac{c_{k-1}}{c_{n}} f\left(\frac{a_{k}}{b_{n+1}}\right)+\sum_{k=1}^{n} \frac{c_{n}-c_{k}}{c_{n}} f\left(\frac{a_{k}}{b_{n+1}}\right) \\
& =\sum_{k=2}^{n} \frac{c_{k-1}}{c_{n}} f\left(\frac{a_{k}}{b_{n+1}}\right)+\sum_{k=1}^{n-1} \frac{c_{n}-c_{k}}{c_{n}} f\left(\frac{a_{k}}{b_{n+1}}\right) \\
& =\sum_{k=1}^{n-1}\left(\frac{c_{k}}{c_{n}} f\left(\frac{a_{k+1}}{b_{n+1}}\right)+\frac{c_{n}-c_{k}}{c_{n}} f\left(\frac{a_{k}}{b_{n+1}}\right)\right) .
\end{aligned}
$$

From (7), by using the convexity of $f$, we get that

$$
\begin{aligned}
\frac{c_{n-1}}{c_{n}} \sum_{k=1}^{n} f\left(\frac{a_{k}}{b_{n+1}}\right) & \geq \sum_{k=1}^{n-1}\left(\frac{c_{k}}{c_{n}} f\left(\frac{a_{k+1}}{b_{n+1}}\right)+\frac{c_{n}-c_{k}}{c_{n}} f\left(\frac{a_{k}}{b_{n+1}}\right)\right) \\
& \geq \sum_{k=1}^{n-1} f\left(\frac{c_{k}}{c_{n}}\left(\frac{a_{k+1}}{b_{n+1}}\right)+\frac{c_{n}-c_{k}}{c_{n}}\left(\frac{a_{k}}{b_{n+1}}\right)\right) .
\end{aligned}
$$

We see now by (G3), because $a_{k}>0, b_{k}>0$ and $c_{k}>0, k \geq 1$, that

$$
\begin{aligned}
\left(\frac{c_{k}}{c_{n}}\left(\frac{a_{k+1}}{b_{n+1}}\right)+\frac{c_{n}-c_{k}}{c_{n}}\left(\frac{a_{k}}{b_{n+1}}\right)\right)-\frac{a_{k}}{b_{n}} & \\
=\frac{a_{k}}{c_{n} b_{n+1}}\left(c_{k}\left(\frac{a_{k+1}}{a_{k}}-1\right)-c_{n}\left(\frac{b_{n+1}}{b_{n}}-1\right)\right) & \geq 0, \\
& 1 \leq k<n .
\end{aligned}
$$

Hence, from (9),

$$
\left(\frac{c_{k}}{c_{n}}\left(\frac{a_{k+1}}{b_{n+1}}\right)+\frac{c_{n}-c_{k}}{c_{n}}\left(\frac{a_{k}}{b_{n+1}}\right)\right) \geq \frac{a_{k}}{b_{n}}, \quad 1 \leq k<n,
$$

and, as $f$ is increasing on the interval $[D, E]$, from (10):

$$
f\left(\frac{c_{k}}{c_{n}}\left(\frac{a_{k+1}}{b_{n+1}}\right)+\frac{c_{n}-c_{k}}{c_{n}}\left(\frac{a_{k}}{b_{n+1}}\right)\right) \geq f\left(\frac{a_{k}}{b_{n}}\right), \quad 1 \leq k<n .
$$

From (7) and (11), we get that

$$
\begin{aligned}
\frac{c_{n-1}}{c_{n}} \sum_{k=1}^{n} f\left(\frac{a_{k}}{b_{n+1}}\right) & \geq f\left(\frac{c_{k}}{c_{n}}\left(\frac{a_{k+1}}{b_{n+1}}\right)+\frac{c_{n}-c_{k}}{c_{n}}\left(\frac{a_{k}}{b_{n+1}}\right)\right) \\
& \geq f\left(\frac{a_{k}}{b_{n}}\right), \quad 2 \leq k<n .
\end{aligned}
$$

From (12) and (4), we get that (3) holds, which means that $A_{n}$ is increasing with $n, n \geq 2$.

\section{References}

[1] S. Abramovich, G. Jameson and G. Sinnamon, Inequalities for averages of convex and superquadratic functions, J. Inequal. Pure Appl. Math. 5 (2004), Article 91.

Also solved by Mihaly Bencze (Romania), Socratis Varelogiannis (France), Alexander Vauth (Germany).

188. For a function $f: \mathbb{R} \rightarrow \mathbb{R}$ and a positive integer $n$, we denote by $f^{n}$ the function defined by $f^{n}(x)=(f(x))^{n}$.

(a) Show that if $f: \mathbb{R} \rightarrow \mathbb{R}$ is a function that has an antiderivative then $f^{n}: \mathbb{R} \rightarrow \mathbb{R}$ satisfies the intermediate value property for any $n \geq 1$.

(b) Give an example of a function $f: \mathbb{R} \rightarrow \mathbb{R}$ that has an antiderivative and for which $f^{n}: \mathbb{R} \rightarrow \mathbb{R}$ has no antiderivatives for any $n \geq 2$.

(Dorin Andrica, Babesş Bolyai University, Cluj-Napoca, Romania, and Vlad Crişan, University of Göttingen, Germany)

\section{Solution by the proposers.}

(a) Since $f$ has an antiderivative, $f$ satisfies the intermediate value property (IVP). The compositum of two functions satisfying the IVP is again a function satisfying IVP. Note that

$$
f^{n}=g \circ f,
$$

where $g: \mathbb{R} \rightarrow \mathbb{R}$ is defined as $g(x)=x^{n}$. It is easy to see that $g$ has the IVP, hence $f^{n}=g \circ f$ must also satisfy the IVP.

(b) We use the following classical result.

Lemma 1 For $a \in \mathbb{R} \backslash\{0\}$ and $b \in \mathbb{R}$, the function defined by

$$
f_{a, b}(x)=\left\{\begin{array}{l}
\cos \frac{a}{x} \text { if } x \neq 0 \\
b \text { if } x=0
\end{array}\right.
$$

has an antiderivative if and only if $b=0$.

From Lemma 1, it follows that the function $f: \mathbb{R} \rightarrow \mathbb{R}$ given by

$$
f(x)= \begin{cases}\cos ^{2} \frac{1}{x} & \text { if } x \neq 0 \\ \frac{1}{2} & \text { if } x=0\end{cases}
$$

has an antiderivative.

We now have that

$$
f^{n}(x)= \begin{cases}\cos ^{2 n} \frac{1}{x} & \text { if } x \neq 0 \\ \left(\frac{1}{2}\right)^{n} & \text { if } x=0\end{cases}
$$

We shall prove that $f^{n}$ has no antiderivative for $n \geq 2$. For this, one uses the following identity, whose proof is just a simple induction.

Lemma 2 For any reals $x_{1}, \ldots, x_{m}$, one has

$$
\cos x_{1} \cos x_{2} \cdots \cos x_{m}=\frac{1}{2^{m}} \sum \cos \left( \pm x_{1} \pm x_{2} \pm \cdots \pm x_{m}\right),
$$

where the sum on the right side is over all $2^{m}$ possible choices of signs. 
Using Lemma 2, we can write

$$
\begin{aligned}
f^{n}(x) & = \begin{cases}\frac{1}{2^{2 n}} \sum_{+,-} \cos (\underbrace{ \pm \frac{1}{x} \pm \cdots \pm \frac{1}{x}}_{2 n \text { terms }}) & \text { if } x \neq 0 \\
\frac{1}{2^{n}} & \text { if } x=0,\end{cases} \\
& =\frac{1}{2^{2 n}} \sum_{+,-\neq 0} f_{(\underbrace{ \pm 1 \pm \cdots \pm 1}_{2 n \text { terms }}, 0)}+ \begin{cases}\frac{1}{2^{2 n}} S_{n} & \text { if } x \neq 0 \\
\frac{1}{2^{n}} & \text { if } x=0,\end{cases}
\end{aligned}
$$

where the last sum is over all possible choices of signs for which we have

$$
\underbrace{ \pm 1 \pm \cdots \pm 1}_{2 n \text { terms }} \neq 0
$$

and $S_{n}$ is the number of combinations for which

$$
\underbrace{ \pm 1 \pm \cdots \pm 1}_{2 n \text { terms }}=0 \text {. }
$$

Using Lemma 1, we have that $f^{n}$ has an antiderivative if and only if $\frac{1}{2^{2 n}} S_{n}=\frac{1}{2^{n}}$, i.e., $S_{n}=2^{n}$. On the other hand, it is clear that

$$
S_{n}=\left(\begin{array}{c}
2 n \\
n
\end{array}\right)
$$

so the last condition becomes $\left(\begin{array}{c}2 n \\ n\end{array}\right)=2^{n}$. This equation has no solution for $n \geq 2$ since, for example, any prime $p$ between $n$ and $2 n$ divides $\left(\begin{array}{c}2 n \\ n\end{array}\right)$ but does not divide $2^{n}$.

Also solved by Mihaly Bencze (Romania), John N. Daras (Greece), Sotirios E. Louridas (Greece).

189

(a) Let $\left\{f_{n}\right\}_{n=1}^{\infty}$ be an increasing sequence of continuous realvalued functions on a compact metric space $X$ that converges pointwisely to a continuous function $f$. Show that the convergence must be uniform.

(b) Show by a counterexample that the compactness of $X$ in (a) is necessary.

(c) Determine whether (a) remains valid if the sequence $\left\{f_{n}\right\}_{n=1}^{\infty}$ is not monotone.

(W. S. Cheung, University of Hong Kong, Pokfulam, Hong Kong)

\section{Solution by the proposer.}

(a) For any $n \in \mathbb{N}$, write $g_{n}:=f-f_{n}$. As $f_{n} \uparrow f$ pointwisely on $X$, we have $g_{n} \downarrow 0$ pointwisely on $X$.

Let $\varepsilon>0$ be given. For any $x \in X$, there exists $N_{x} \in \mathbb{N}$ such that

$$
g_{N_{x}}(x)<\varepsilon .
$$

Since $g_{N_{x}}$ is continuous, there exists an open neighbourhood $B_{x}$ of $x$ in $X$ such that

$$
g_{N_{x}}(y)<\varepsilon \quad \text { for all } y \in B_{x} .
$$

Do this for every $x \in X$. The open cover $\left\{B_{x}: x \in X\right\}$ of $X$ has a finite subcover, say $\left\{B_{x_{1}}, \ldots, B_{x_{k}}\right\}$. Write

$$
N:=\max \left\{N_{x_{1}}, \ldots, N_{x_{k}}\right\} \text {. }
$$

For any $y \in X$, there is an $i \in\{1, \ldots, k\}$ such that $y \in B_{x_{i}}$. Hence, for any $n \geq N \geq N_{x_{i}}$, we have

$$
0 \leq g_{n}(y) \leq g_{N}(y) \leq g_{N_{x_{i}}}(y)<\varepsilon .
$$

Hence $\left\{g_{n}\right\} \rightarrow 0$ uniformly on $X$ and so $\left\{f_{n}\right\} \rightarrow f$ uniformly on $X$. (b) For any $n \in \mathbb{N}$, consider $f_{n}: \mathbb{R} \rightarrow \mathbb{R}$ given by

$$
f_{n}(x):=\left(1-\frac{1}{n}\right) x \text {. }
$$

Clearly, $f_{n}(x) \uparrow f(x):=x$ pointwisely on $\mathbb{R}$ but, as

$$
\sup _{n \rightarrow \infty}\left\{\left|f_{n}(x)-f(x)\right|: x \in \mathbb{R}\right\}=\sup _{n \rightarrow \infty}\left\{\frac{|x|}{n}: x \in \mathbb{R}\right\}=\infty,
$$

the convergence is not uniform.

(c) Without the monotonicity, (a) will no longer be valid. For example, consider the sequence $f_{n}: X=[0,1] \rightarrow \mathbb{R}$ given by

$$
f_{n}(x):= \begin{cases}0 & 0 \leq x \leq 1-\frac{2}{n} \\ n^{2} x-n^{2}+2 n & 1-\frac{2}{n} \leq x \leq 1-\frac{1}{n} \\ -n^{2} x+n^{2} & 1-\frac{1}{n} \leq x \leq 1 .\end{cases}
$$

$f_{n}$ is continuous on $X$ for each $n$ and $f_{n} \rightarrow f \equiv 0$ pointwisely on $X$ but, as

$\sup _{n \rightarrow \infty}\left\{\left|f_{n}(x)-f(x)\right|: x \in X\right\}=\sup _{n \rightarrow \infty}\left\{f_{n}(x): x \in X\right\}=\sup _{n \rightarrow \infty}\{n\}=\infty$, the convergence is not uniform.

Also solved by Socratis Varelogiannis (France), Alexander Vauth (Germany), Jeff Webb (UK).

190. Let $\left\{a_{n}\right\}$ be a sequence of positive numbers. In the ratio test, we know that the condition

$$
\lim _{n \rightarrow \infty} \frac{a_{n+1}}{a_{n}}=1
$$

is not sufficient to determine whether the series $\sum_{n=1}^{\infty} a_{n}$ is convergent or divergent. For example, if $a_{n}=1 / n$ then

$$
\frac{a_{n+1}}{a_{n}}=\frac{n}{n+1}=1-\frac{1}{n+1}=1-\frac{n+1}{(n+1)^{2}}
$$

and if $a_{n}=1 / n^{2}$ then

$$
\frac{a_{n+1}}{a_{n}}=\frac{n^{2}}{(n+1)^{2}}=1-\frac{2 n+1}{(n+1)^{2}} .
$$

Hence, the coefficient $a$ in the expression $1-\frac{a n+1}{(n+1)^{2}}$ plays an important role in the convergence of $\sum a_{n}$. In this question, we would like to study it more closely.

Let $a$ be a non-negative real number and let $\left\{a_{n}\right\}$ be a sequence with $a_{n}>0$, satisfying

$$
\frac{a_{n+1}}{a_{n}} \leq 1-\frac{a n+1}{(n+1)^{2}}
$$

for all $n \geq n_{0}:=[|2-a|]+1$, where $[x]$ is the integral part of $x$.

(i) Show that if $a>0$ then

$$
\lim _{n \rightarrow \infty} a_{n}=0 .
$$

If $a=0$, for any $\lambda>0$, find an example such that

$$
\lim _{n \rightarrow \infty} a_{n}=\lambda .
$$

(ii) Show that if $a>1$ then

$$
\sum_{n=1}^{\infty} a_{n}
$$

is convergent. Is this still true when $a=1$ ?

(Stephen Choi and Peter Lam, Simon Fraser University, Burnaby B.C., Canada) 
Solution by the proposers. (i) In view of (13), the sequence $\left\{a_{n}\right\}$ is eventually monotonically decreasing and bounded below by 0 . So, $\lim _{n \rightarrow \infty} a_{n}$ exists and is non-negative.

By (13), we have

$$
a_{n+1} \leq\left(\frac{n^{2}+(2-a) n}{(n+1)^{2}}\right) a_{n} \leq \cdots \leq a_{n_{0}} \prod_{i=n_{0}}^{n}\left(\frac{i^{2}+(2-a) i}{(i+1)^{2}}\right)
$$

for all $n \geq n_{0}$. It follows that

$$
\frac{1}{a_{n+1}} \geq \frac{1}{a_{n_{0}}} \prod_{i=n_{0}}^{n}\left(\frac{(i+1)^{2}}{i^{2}+(2-a) i}\right)=\frac{1}{a_{n_{0}}} \prod_{i=n_{0}}^{n}\left(1+\frac{a i+1}{i^{2}+(2-a) i}\right)
$$

for all $n \geq n_{0}$.

If $a>2$ then

$$
\sum_{i=n_{0}}^{\infty} \frac{a i+1}{i^{2}+(2-a) i} \geq \sum_{i=n_{0}}^{\infty} \frac{a}{i}=\infty .
$$

If $2>a>0$ then

$$
\sum_{i=n_{0}}^{\infty} \frac{a i+1}{i^{2}+(2-a) i} \geq \sum_{i=n_{0}}^{\infty} \frac{a}{2 i}=\infty
$$

because $i>(2-a)$ for $i \geq n_{0}$. Since

$$
\prod_{i=n_{0}}^{n}\left(1+\frac{a i+1}{i^{2}+(2-a) i}\right) \geq 1+\sum_{n=n_{0}}^{n} \frac{a i+1}{i^{2}+(2-a) i},
$$

we have $\lim _{n \rightarrow \infty} a_{n}=0$ by (15).

If $a=0$, for any $\lambda>0$, take $a_{n}=\lambda(n+1) / n$. Then,

$$
\frac{a_{n+1}}{a_{n}}=1-\frac{1}{(n+1)^{2}}
$$

and $\lim _{n \rightarrow \infty} a_{n}=\lambda$.

(ii) If $a>1$, in view of (14), for any $n \geq n_{0}$, we have

$$
\begin{aligned}
a_{n+1} & \leq a_{n_{0}} \prod_{i=n_{0}}^{n}\left(\frac{i^{2}+(2-a) i}{(i+1)^{2}}\right) \\
& =a_{n_{0}}\left(\prod_{i=n_{0}}^{n} \frac{i}{i+1}\right) \prod_{i=n_{0}}^{n}\left(\frac{i+(2-a)}{i+1}\right) \\
& =a_{n_{0}} \frac{n_{0}}{n+1} \prod_{i=n_{0}}^{n}\left(1-\frac{a-1}{i+1}\right) \\
& =\frac{a_{n_{0}} n_{0}}{n+1} \exp \left\{\sum_{i=n_{0}}^{n} \log \left(1-\frac{a-1}{i+1}\right)\right\} .
\end{aligned}
$$

Now, using an elementary inequality $\log (1-x) \leq-x$ for $0 \leq x<1$, we have

$$
\begin{aligned}
a_{n+1} & \leq \frac{a_{n_{0}} n_{0}}{n+1} \exp \left\{-(a-1) \sum_{i=n_{0}}^{n} \frac{1}{i+1}\right\} \\
& \leq \frac{a_{n_{0}} n_{0}}{n+1} \exp \left\{-(a-1) \int_{n_{0}+1}^{n+2} \frac{1}{x} d x\right\} \\
& \leq \frac{a_{n_{0}} n_{0}}{n+1} \exp \left\{-(a-1) \log \left(\frac{n+2}{n_{0}+1}\right)\right\} \\
& =\frac{a_{n_{0}} n_{0}}{n+1}\left(\frac{n_{0}+1}{n+2}\right)^{(a-1)} \\
& \leq \frac{a_{n_{0}}\left(n_{0}+1\right)^{a}}{(n+1)^{a}}
\end{aligned}
$$

for all $n \geq n_{0}$. Since the series $\sum_{n=1}^{\infty} \frac{1}{(n+1)^{a}}$ converges, so does $\sum_{n=1}^{\infty} a_{n}$.
If $a=1$, the statement is false by considering the counterexample $a_{n}=1 / n$.

Also solved by Mihaly Bencze (Romania), Panagiotis Krasopoulos (Greece).

191. Show that for any $a, b>0$, we have

$$
\frac{1}{2}\left(1-\frac{\min \{a, b\}}{\max \{a, b\}}\right)^{2} \leq \frac{b-a}{a}-\ln b+\ln a \leq \frac{1}{2}\left(\frac{\max \{a, b\}}{\min \{a, b\}}-1\right)^{2} .
$$

(Silvestru Sever Dragomir, Victoria University, Melbourne City, Australia)

Solution by the proposer. Integrating by parts, we have

$$
\int_{a}^{b} \frac{b-t}{t^{2}} d t=\frac{b-a}{a}-\ln b+\ln a
$$

for any $a, b>0$.

If $b>a$ then

$$
\frac{1}{2} \frac{(b-a)^{2}}{a^{2}} \geq \int_{a}^{b} \frac{b-t}{t^{2}} d t \geq \frac{1}{2} \frac{(b-a)^{2}}{b^{2}} .
$$

If $a>b$ then

$$
\int_{a}^{b} \frac{b-t}{t^{2}} d t=-\int_{b}^{a} \frac{b-t}{t^{2}} d t=\int_{b}^{a} \frac{t-b}{t^{2}} d t
$$

and

$$
\frac{1}{2} \frac{(b-a)^{2}}{b^{2}} \geq \int_{b}^{a} \frac{t-b}{t^{2}} d t \geq \frac{1}{2} \frac{(b-a)^{2}}{a^{2}} .
$$

Therefore, by (17) and (18), we have for any $a, b>0$ that

$$
\int_{a}^{b} \frac{b-t}{t^{2}} d t \geq \frac{1}{2} \frac{(b-a)^{2}}{\max ^{2}\{a, b\}}=\frac{1}{2}\left(\frac{\min \{a, b\}}{\max \{a, b\}}-1\right)^{2}
$$

and

$$
\int_{a}^{b} \frac{b-t}{t^{2}} d t \leq \frac{1}{2} \frac{(b-a)^{2}}{\min ^{2}\{a, b\}}=\frac{1}{2}\left(\frac{\max \{a, b\}}{\min \{a, b\}}-1\right)^{2} .
$$

By the representation (16), we then get the desired result.

Also solved by Jim K. Kelesis (Greece), Panagiotis Krasopoulos (Greece), Alexander Vauth (Germany).

192. Let $a, b, c, d \in \mathbb{R}$ with $b c>0$. Calculate

$$
\lim _{n \rightarrow \infty}\left(\begin{array}{cc}
\cos \frac{a}{n} & \sin \frac{b}{n} \\
\frac{c}{n} & \cos \frac{d}{n}
\end{array}\right)^{n}
$$

(Ovidiu Furdui, Technical University of Cluj-Napoca, Cluj-Napoca, Romania) 
Solution by the proposer. The limit equals

$$
\left(\begin{array}{cc}
\cosh \sqrt{b c} & \frac{b}{\sqrt{b c}} \sinh \sqrt{b c} \\
\frac{c}{\sqrt{b c}} \sinh \sqrt{b c} & \cosh \sqrt{b c}
\end{array}\right)
$$

Let

$$
A=\left(\begin{array}{cc}
\cos \frac{a}{n} & \sin \frac{b}{n} \\
\frac{c}{n} & \cos \frac{d}{n}
\end{array}\right) .
$$

The characteristic equation of $A$ is given by

$$
\lambda^{2}-\left(\cos \frac{a}{n}+\cos \frac{d}{n}\right) \lambda+\cos \frac{a}{n} \cos \frac{d}{n}-\frac{c}{n} \sin \frac{b}{n}=0 .
$$

The discriminant of this equation is $\Delta=\left(\cos \frac{a}{n}-\cos \frac{d}{n}\right)^{2}+\frac{4 c}{n} \sin \frac{b}{n}$. Since $b c>0$, one has that either both $b$ and $c$ are positive or both are negative real numbers. If $b$ and $c$ are positive numbers, one has that for large $n, 0<\frac{b}{n}<\pi$ and hence $\Delta>0$. If $b$ and $c$ are negative real numbers then, for large $n$, one has that $-\pi<\frac{b}{n}<0$ and it follows, since $\sin \frac{b}{n}<0$, that $\Delta>0$. Therefore, there are two real distinct eigenvalues of $A$ given by

$$
\begin{aligned}
& \lambda_{1}=\frac{1}{2}\left(\cos \frac{a}{n}+\cos \frac{d}{n}\right)+\frac{1}{2} \sqrt{\left(\cos \frac{a}{n}-\cos \frac{d}{n}\right)^{2}+\frac{4 c}{n} \sin \frac{b}{n}}, \\
& \lambda_{2}=\frac{1}{2}\left(\cos \frac{a}{n}+\cos \frac{d}{n}\right)-\frac{1}{2} \sqrt{\left(\cos \frac{a}{n}-\cos \frac{d}{n}\right)^{2}+\frac{4 c}{n} \sin \frac{b}{n}} .
\end{aligned}
$$

Now we need Theorem 4.7 on page 194 (see also Remark 3.1 on page 109) in [1], which states that if $n \in \mathbb{N}, A \in \mathcal{M}_{2}(\mathbb{C})$ and $\lambda_{1} \neq \lambda_{2}$ are the eigenvalues of $A$ then

$$
A^{n}=\frac{\lambda_{1}^{n}-\lambda_{2}^{n}}{\lambda_{1}-\lambda_{2}} A+\frac{\lambda_{1} \lambda_{2}^{n}-\lambda_{2} \lambda_{1}^{n}}{\lambda_{1}-\lambda_{2}} I_{2}
$$

Let $k \in \mathbb{N}$. An easy calculation, based on the previous formula, shows that

$$
A^{k}=\frac{\lambda_{1}^{k}\left(1-\lambda_{2}\right)+\lambda_{2}^{k}\left(\lambda_{1}-1\right)}{\lambda_{1}-\lambda_{2}} I_{2}+\frac{\lambda_{1}^{k}-\lambda_{2}^{k}}{\lambda_{1}-\lambda_{2}}\left(\begin{array}{cc}
\cos \frac{a}{n}-1 & \sin \frac{b}{n} \\
\frac{c}{n} & \cos \frac{d}{n}-1
\end{array}\right) .
$$

When $k=n$, one has that

$$
\begin{aligned}
A^{n}=\frac{\lambda_{1}^{n}\left(1-\lambda_{2}\right)+\lambda_{2}^{n}\left(\lambda_{1}-1\right)}{\lambda_{1}-\lambda_{2}} & \\
& +\frac{\lambda_{1}^{n}-\lambda_{2}^{n}}{\lambda_{1}-\lambda_{2}}\left(\begin{array}{cc}
\cos \frac{a}{n}-1 & \sin \frac{b}{n} \\
\frac{c}{n} & \cos \frac{d}{n}-1
\end{array}\right) .
\end{aligned}
$$

We have that $\lim _{n \rightarrow \infty} \lambda_{1}^{n}=\mathrm{e}^{\sqrt{b c}}$ and $\lim _{n \rightarrow \infty} \lambda_{2}^{n}=\mathrm{e}^{-\sqrt{b c}}$. On the other hand, a calculation shows that $\lim _{n \rightarrow \infty} \frac{1-\lambda_{2}}{\lambda_{1}-\lambda_{2}}=\lim _{n \rightarrow \infty} \frac{\lambda_{1}-1}{\lambda_{1}-\lambda_{2}}=\frac{1}{2}, \lim _{n \rightarrow \infty} \frac{\cos \frac{a}{n}-1}{\lambda_{1}-\lambda_{2}}=$ $\lim _{n \rightarrow \infty} \frac{\cos \frac{d}{n}-1}{\lambda_{1}-\lambda_{2}}=0, \lim _{n \rightarrow \infty} \frac{\sin \frac{b}{n}}{\lambda_{1}-\lambda_{2}}=\frac{b}{2 \sqrt{b c}}$ and $\lim _{n \rightarrow \infty} \frac{\frac{c}{n}}{\lambda_{1}-\lambda_{2}}=\frac{c}{2 \sqrt{b c}}$.

Passing to the limit as $n \rightarrow \infty$ in (19) and using the previous limits we have that

$$
\begin{aligned}
\lim _{n \rightarrow \infty} A^{n} & =\frac{\mathrm{e}^{\sqrt{b c}}+\mathrm{e}^{-\sqrt{b c}}}{2} I_{2}+\left(\mathrm{e}^{\sqrt{b c}}-\mathrm{e}^{-\sqrt{b c}}\right)\left(\begin{array}{cc}
0 & \frac{b}{2 \sqrt{b c}} \\
\frac{c}{2 \sqrt{b c}} & 0
\end{array}\right) \\
& =\left(\begin{array}{cc}
\cosh \sqrt{b c} & \frac{b}{\sqrt{b c}} \sinh \sqrt{b c} \\
\frac{c}{\sqrt{b c}} \sinh \sqrt{b c} & \cosh \sqrt{b c}
\end{array}\right)
\end{aligned}
$$

and the problem is solved.

\section{References}

[1] Vasile Pop, Ovidiu Furdui, Square Matrices of Order 2. Theory, Applications and Problems. Cham, Springer, 2017.

Also solved by Mihaly Bencze (Romania), Jim K. Kelesis (Greece), Sotirios E. Louridas (Greece), Julio Cesar Mohnsam (Brazil), Socratis Varelogiannis (France).

We would like you to submit solutions to the proposed problems and ideas on the open problems. Send your solutions by email to Michael Th. Rassias, Institute of Mathematics, University of Zürich, Switzerland, michail.rassias@math.uzh.ch.

We also solicit your new problems with their solutions for the next "Solved and Unsolved Problems" column, which will be devoted to topology. 